\title{
Detection of Circulating Dengue Virus Serotypes in a Tertiary Care Centre in Central Kerala, 2016
}

\author{
Kavitha R. Nair ${ }^{1}$, Seema Oommen ${ }^{1}$, O.A. Jagan $^{3}$ and Vidya Pai $^{2}$ \\ ${ }^{1}$ Department of Microbiology, Pushpagiri Institute of Medical Sciences and Research Centre, \\ Tiruvalla, Kerala, India \\ ${ }^{2}$ Department of Microbiology, Yenepoya Medical College, Yenepoya University, Mangalore, \\ Karnataka, India \\ ${ }^{3}$ Department of Virology, Amrita Institute of Medical Sciences and Research Centre, Kochi, \\ Kerala, India \\ *Corresponding author:
}

\begin{tabular}{|l|}
\hline Ke y w o r d s \\
Dengue, Dengue \\
serotypes, Co- \\
infecting dengue \\
serotypes, DENV 1- \\
4 \\
\hline Article Info \\
\hline $\begin{array}{l}\text { Accepted: } \\
\text { 17 December } 2018 \\
\text { Available Online: } \\
\text { 10 January } 2019\end{array}$ \\
\hline
\end{tabular}

A B S T R A C T

Dengue is the most common mosquito-borne viral infection in tropical and sub-tropical countries. Dengue virus, a single stranded positive sense RNA virus belongs to the genus Flavivirus in the family Flaviviridae. Both Aedes aegypti and Aedes albopictus are the main vectors for dengue virus in India. Dengue illnesses are caused by one or more of the serologically related viruses namely DENV-1, DENV-2, DENV-3, DENV-4 and the newly identified DENV-5.India has faced a dramatically expanded dengue virus transmission over the last few decades, with rapidly changing epidemiology. In recent years, India has reported increased incidences of concurrent infection with multiple serotypes of dengue viruses (DENV). In the present study, we have identified the circulating dengue virus serotypes in a tertiary care centre in Central Kerala in 2016. A prospective study was conducted in the Department of Microbiology, of a tertiary care centre in Central Kerala from January 2016 to December 2016. A total of 274 adult patients whose serum samples were NS1 positive were further subjected to conventional multiplex RT- PCR. Out of 274, Dengue RT PCR was positive for 159 (58\%) samples. Of 159, 64(40.3\%) were identified as DENV-1, 55 (34.6\%) DENV-2, seven (4.4\%) DENV-3, two (1.25\%) were DENV-4. Twenty- two cases (13.8\%) were co-infected with DENV- 1 and DENV-2, six (3.77\%) with DENV-2 and DENV-4 and three (1.88\%) with DENV-2 and DENV-3. All the four dengue serotypes circulated in Kerala in the year 2016, of which DENV-1 was found to be the predominant serotype followed by DENV-2.

\section{Introduction}

Dengue is an arthropod borne Flavivirus comprising four distinct serotypes namely DENV-1, DENV-2, DENV-3, DENV-4 and the newly identified DENV-5 (Mustafa et al., 2015). They are mainly transmitted by Aedes aegypti and Aedes albopticus. Infection by one serotype induces life-long immunity against re-infection by the same serotype, but only 
transient and partial protection against infection with the other serotypes (McBride et al., 2000). Dengue infection ranges from subclinical infection to mild self-limiting disease to life threatening conditions like dengue hemorrhagic fever (DHF)/dengue shock syndrome (DSS). An estimated 2.5 billion people are living in the areas under risk for epidemic dengue virus transmission. Globally, one hundred million cases of dengue fever (DF) and 450,000 cases of dengue hemorrhagic fever/dengue shock syndrome (DHF/DSS) are reported annually (WHO, 2009).

Dengue virus is a positive-stranded enveloped RNA virus and is composed of three structural protein genes, which encode the nucleocapsid or core (C) protein, a membrane-associated (M) protein, an enveloped (E) glycoprotein and seven nonstructural (NS) proteins. Each serotype has unique characteristics and can present with varying clinical manifestations, in a population depending upon its interaction with the host response (McBride et al., 2000). Laboratory diagnosis of dengue infection is primarily achieved through sero-diagnosis and molecular assays for detection of viral RNA and rarely virus isolation from acute phase serum (Guzman et al., 1996).

Early diagnosis of dengue is crucial in the absence of any licensed antiviral therapy and prophylaxis. Serological assays are most commonly used for diagnosis of dengue infection since they are relatively inexpensive and easy to perform. The diagnosis is achieved serologically by detecting dengue-specific $\mathrm{IgM}$ and $\mathrm{IgG}$ antibodies, which generally appear 7-8 days after the onset of illness (Gubler et al., 1991). The detection of $\mathrm{IgG}$, due to cross reactivity with other closely related members of Flaviviruses, needs to be confirmed with paired sera, which is not practical in most cases (WHO Dengue Bulletin, 2003) NS1 (Non-Structural protein-
1) is a glycoprotein produced by Flaviviruses. NS1 exists as a monomer, a dimer (membrane bound protein, mNS1) and a hexamer (secreted protein, sNS1). The intracellular NS1 is mainly implicated in viral replication and viability, whereas the secreted and membrane-bound NS1 have been reported to elicit the immune response. There are regions of similarity and dissimilarity among the NS1 sequences of different Flaviviruses (Rastogi et al., 2016). Because this protein is secreted into the bloodstream, NS1 antigen detection using capture ELISA can be used to detect the presence of acute dengue infection especially in the first seven days of infection. In dengue, secondary infection with a heterologous serotype often leads to severe clinical manifestations like dengue haemorrhagic fever (DHF) and dengue shock syndrome (DSS) (McBride et al., 2000).

Virus isolation though considered as the 'gold standard', it is technically demanding and time consuming. The molecular methods based on PCR technique offers a rapid assay for detecting and typing dengue virus and should be done within five days after the onset of symptoms. The RT-PCR targeting the conserved regions of $C p r M$ gene junction is widely employed for precise confirmation of an infection (Lanciotti et al., 1992).

The first confirmed report of dengue infection in India dates back to 1940 s and thereafter several states began to report the disease which mostly struck in epidemic proportions often inflicting heavy morbidity and mortality, both in urban and rural environments (Lall et al., 1996). Dengue virus infection has been prevalent in India for the last 50 years (Chakravarti et al., 2012). Like Southeast Asia, the region has become hyperendemic to dengue with the circulation of all the four serotypes (Weaver et al., 2009). In Kerala cases of dengue with some deaths were reported in 1997 for the first time (Kalra et al., 
2004). The first documentation of dengue happened in Kerala 44 years ago. Since then the infection remained a low-profile disease in the state till its re-emergence $\mathrm{n}$ the epidemic form causing significant morbidity and mortality in 2003 (Tyagi et al., 2006).

Dengue fever in Kerala has shown an increasing trend since 2006. Topographically, Pathanamthitta district is a semi-forested region with hills and hillocks located in the foothills of the Western Ghats. Distribution of serotypes has found to be an important indicator for the severity of the disease. In the present study, we have attempted to identify the circulating dengue virus serotypes in a tertiary care centre in Central Kerala in 2016.

\section{Materials and Methods}

\section{Serum samples}

Blood samples collected from adult patients with acute fever admitted to a tertiary care centre in Central Kerala for a period of one year (January to December, 2016) were included the study. Ethical clearance dated 29th October 2015, (PIMSRC/E1/388A/45/2015) was obtained from the Institutional Ethical Committee. Informed consent from patients for characterization of the viral isolates was obtained. NS1(Non Structural-1)antigen [Panbio Dengue NS1capture Enzyme Linked ImmunoSorbent Assay (ELISA)] and dengue IgM and IgG antibody (Panbio Dengue IgM Capture ELISA, Panbio Dengue IgG Capture ELISA) were tested for all samples suspected to be dengue.

A total of 274 patients who were 18 years of age or above and tested positive for NS1 dengue antigen were subjected to dengue serotyping by conventional multiplex reverse transcriptase (RT)-PCR. Dengue cases were classified as primary dengue and secondary dengue based on the Panbio $\operatorname{IgM} / \operatorname{IgG}$ ratios. Values $<1.2$ were considered as secondary dengue and values $>1.2$ as primary dengue (Guzman et al., 2010).

\section{Viral RNA extraction}

Blood serum separated from these samples was processed using a viral RNA extraction kit (Qiagen). Approximately, $150 \mu 1$ serum samples were used for extraction of viral RNA following the manufacturer's protocol. After the incubation step for lysis, the samples were passed through Qiagen columns and the viral RNA bound to silica was eluted in $30 \mu 1$ deionized RNase- and DNase-free water after two washing steps. The RNA extracts were stored at $-70{ }^{\circ} \mathrm{C}$ until further processing.

\section{cDNA synthesis and PCR amplification}

A One Step RTPCR kit (OrionXOnestep RTPCR mix) was used for amplification of arboviral diagnostic fragments. The enzyme mix permitted the reaction to be carried out in a single step, using the same buffer for both cDNA synthesis and PCR amplification. Target amplification was the capsid premembrane CprM gene ( 511 bp) using the DNA primers, which are used to check for dengue infection status (Lancoitti et al., 1992). Following this, the protocol of the modified version was used for detection of DENV serotypes (Lanciotti et al., 1997). The primers used for the amplification were as follows:

DEN:

TCAATATGCTGAACGCGCGAGAAACCG DEN1:

CTGGTTCCGTCTCAGTGATCCGGGGG DEN2: AACGCCACAAGGGCCATGAACA DEN3:

TGCTGGTAACATCATCATGAGACAGAG CG

DEN4:

CTCTGTTGTCTTAAACAAGAGAGGTC 
Briefly, the protocol was as follows: reverse transcription at $50^{\circ} \mathrm{C}$ for $30 \mathrm{~min}$, an initial denaturation step at $95^{\circ} \mathrm{C}$ for $15 \mathrm{~min}$, followed by 35 cycles of denaturation at $94^{\circ} \mathrm{C}$ for 30 sec, annealing at $60^{\circ} \mathrm{C}$ for $1 \mathrm{~min}$ and extension at $68^{\circ} \mathrm{C}$ for $1 \mathrm{~min}$. A final extension follows at $68^{\circ} \mathrm{C}$ for $10 \mathrm{~min}$ and finally termination at $4^{\circ} \mathrm{C}$.

The amplicon was subjected to SYBR safe stained $2.0 \%$ agarose gel electrophoresis and observed under a gel documentation system. The size of different bands was compared with a standard marker for the identification of dengue serotypes (DENV-1 489 bp, DENV-2123 bp, DENV-3- 296 bp and DENV-4 - 395 bp (Figure 1).

Patients were classified as primary and secondary dengue by $\operatorname{IgM} / \mathrm{IgG}$ ratio (Guzman et al., 2010). The results of PCR and ELISA were compiled and statistically analysed. A p value less than 0.05 were considered significant.

\section{Results and Discussion}

A total of 274 NS1 antigen positive serum samples were collected during the one year study period (January -December 2016). The highest number of positive cases was recorded during the month of June followed by May and July of 2016.

The present study documents the circulation of all the four serotypes in 2016. Out of 274, Dengue RT-PCR was positive for 159 (58\%) samples. Of 159, 64(40.3\%) were identified as DENV-1 and 55 (34.6\%) as DENV-2, seven $(4.4 \%)$ as DENV-3, two (1.25\%) as DENV-4. Twenty-two cases $(13.8 \%)$ were co-infected with DENV-1 and DENV-2, six (3.77\%) with DENV-2 and DENV-4 and three (1.88\%) with DENV-2 and DENV-3. Patients were classified as primary and secondary dengue based on $\mathrm{IgM} / \mathrm{IgG}$ ratio (tested by PanBio
Dengue $\operatorname{IgM}$ and IgG ELISA). Out of 274 cases, $208(76 \%)$ were identified as primary dengue and $66(24 \%)$ as secondary dengue by using the $\operatorname{IgM} / \mathrm{IgG}$ ratio.

Among the wide spectrum of mild and severe clinical and hemorrhagic manifestations analyzed, there was an increased incidence of gastrointestinal manifestations, namely, abdominal pain in five (9\%), vomiting in $18(32.7 \%)$ and diarrhea in $15(27.2 \%)$ cases and these were more observed in patients infected with DENV-2 than DENV-1. Headache $37(67.2 \%)$ was more observed in DENV-2 cases while two cases $(3.6 \%)$ had bleeding manifestations, were reported only in DENV-2 cases. Statistically there is no significant difference between the serotypes involved, their clinical manifestations and also thrombocytopenia. No mortality cases were reported in the study population. All were symptomatically better and stable at the time of discharge.

Globally dengue transmission has expanded in recent years and all the four dengue virus serotypes (DENV 1-4) are now circulating in Asia, Africa and the Americas, a dramatically different scenario from that which prevailed 20 or 30 years ago, showing almost 20-30-fold increase in the number of dengue hemorrhagic fever cases presently (Gibbons RV et al., 2002). In India, expansion of dengue infections with increasing frequency and severity with circulation of multiple serotypes has been reported. There is also an increased incidence of fatal DHF and DSS, which requires urgent medical intervention (Guzman et al., 2010).

A major outbreak of dengue fever occurred in Kerala during the year 2003. Since then Kerala state continues to have disease outbreaks during the pre-monsoon season (May-July). In recent years, Kerala has witnessed an increased outbreak of two major 
mosquito borne illnesses, Chikungunya (2006) and Dengue fever (2003) (Sreekumar et al, 2010; Tyagi et al., 2006).

In the present study, seasonality of transmission of dengue with increased activity in the pre- monsoon and monsoon season (May-July) was observed and the highest number of cases was recorded in the month of June followed by May and July of 2016. A maximum of $87(31.75 \%)$ samples were tested positive for NS1 antigen in June, followed by May and July where 59 (21.5\%)and $50(18.2 \%)$ cases respectively were positive. These findings correlated with the findings of studies conducted in Kerala, reported highest number of cases in May-July seasons (Kumar et al., 2013). Another study conducted in Udupi, Karnataka also reported highest number of cases in pre-monsoon and monsoon season (Kumar et al., 2010). Thus, the correlation between occurrence of dengue and monsoon season is clearly evident in these studies and is further supported by similar findings from Ludhiana and Karachi (Lal et al., 2007; Khan et al., 2007). Pre-monsoon increase in the number of cases, which could be explained by the stagnation of water after a few bouts of pre-monsoon rainfall and due to high humidity after rainy season, which facilitate vector breeding. These findings indicate that preventive measures against dengue infection should come into full swing before and during water stagnation periods after the initial bouts of rainfall and at the end of monsoon. A study conducted in Mumbai has reported maximum number of dengue reactive cases was seen from the month of October to December with a peak in November, indicating a seasonal trend of the outbreak in the post-monsoon period (Shantha Shubra Das et al., 2016).

The most challenging problem with patient management in dengue infection is rapid diagnosis. Although the commercially available ELISAs offer improved results for the diagnosis, they do not offer serotype specific diagnosis. Serological diagnosis based on detection of $\operatorname{IgM}$ antibodies can be achieved only after 5-7 days of illness. Assays based on NS1 antigen detection claim to provide an early diagnosis of dengue within first seven days of illness. The detection of serotypes causing concurrent infections can be made by virus isolation in cell culture followed by indirect (IFA) using serotype specific monoclonal antibodies and/or Reverse Transcriptase (RT)-PCR. RT-PCR provides an accurate and easy technique which gives serotype specific diagnosis of various circulating dengue viruses and information about co-circulation of different subtypes. In this study the samples that were NS1 positive and received within seven days of illness were tested by RT-PCR for detection of dengue virus RNA (Guzman et al., 1996) (Fig. 2).

Dengue has emerged as the most common arboviral infection with varying clinical manifestations in India. Since the first epidemic in Kolkata during 1963-64, many places in India have been experiencing dengue infections (Bandyopadhyay et al., 1996). One of the largest outbreaks in North India occurred in Delhi and adjoining areas in 1996. The 1996 epidemic was mainly due to DENV2 virus (Dar et al., 1999). Following this, in the post epidemic period in 1997, DENV-1 virus activity was seen in Delhi. Since then Delhi has been known for its endemicity of dengue. Many studies have been conducted to record the variation of the serotypes involved. In 2003, another outbreak occurred in Delhi and Gwalior and DENV-3 was reported to be the predominant serotype involved (Dash et al., 2005). During an outbreak in Delhi in 2006, DENV-3 was the most common etiologic agent followed by DENV-1. All the four dengue virus serotypes were found to cocirculate in the outbreak of 2006 (Bharaj et al., 2008) (Table 1 and 2). 
Table.1 Circulating serotypes and its distribution in primary and secondary dengue

\begin{tabular}{|l|c|c|c|}
\hline \multicolumn{1}{|c|}{ Dengue serotypes } & $\begin{array}{c}\text { No. of positive cases, } \\
\mathbf{n = 2 7 4}\end{array}$ & $\begin{array}{c}\text { Primary } \\
\mathbf{n = 2 0 8}\end{array}$ & $\begin{array}{c}\text { Secondary } \\
\mathbf{n = 6 6}\end{array}$ \\
\hline PCR positives & $159(\mathbf{5 8 \%})$ & $109(\mathbf{5 2 \%})$ & $50(\mathbf{7 6 \%})$ \\
\hline DENV-1 & $64(\mathbf{4 0 . 3 \% )}$ & $44(\mathbf{4 0 . 3 \%})$ & $20(\mathbf{4 0 \%})$ \\
\hline DENV-2 & $55(\mathbf{3 4 . 6 \%})$ & $37(\mathbf{3 4 \%})$ & $18(\mathbf{3 6 \%})$ \\
\hline DENV-3 & $7(\mathbf{4 . 4 \%})$ & $5(\mathbf{4 . 5 \%})$ & $2(\mathbf{4 \%})$ \\
\hline DENV-4 & $2(\mathbf{1 . 2 5} \%)$ & $2(\mathbf{1 . 8 3 \%})$ & - \\
\hline DENV-1\&DENV-2 & $22(\mathbf{1 3 . 8 \%})$ & $14(\mathbf{1 2 . 8 \%})$ & $\mathbf{8}(\mathbf{1 6 \%})$ \\
\hline DENV-2\&DENV-4 & $6(\mathbf{3 . 7 7 \%})$ & $4(\mathbf{3 . 6 \%})$ & $\mathbf{2}(\mathbf{4} \%)$ \\
\hline DENV-2 \&DENV-3 & $3(\mathbf{1 . 8 8} \%)$ & $3(\mathbf{2 . 8 \%})$ & - \\
\hline
\end{tabular}

Table.2 Correlation of clinical features and complications with detected serotypes

\begin{tabular}{|c|c|c|c|c|c|}
\hline Dengue serotypes & $\begin{array}{c}\text { DENV-1 } \\
\mathrm{N}=64\end{array}$ & $\begin{array}{c}\text { DENV-2 } \\
\mathrm{N}=55\end{array}$ & $\begin{array}{l}\text { DENV-3 } \\
\text { N=7 }\end{array}$ & $\begin{array}{c}\text { DENV-4 } \\
\mathrm{N}=2\end{array}$ & p value \\
\hline Headache, n (\%) & $33(51.5 \%)$ & $37(67.2 \%)$ & $5(71.4 \%)$ & $1(50 \%)$ & 0.647 \\
\hline Retro-orbital pain, n (\%) & $5(7.8 \%)$ & $6(10.9 \%)$ & - & $1(50 \%)$ & 0.261 \\
\hline Myalgia, n (\%) & $42(65.6 \%)$ & $38(69 \%)$ & $5(71.4 \%)$ & $2(100 \%)$ & 0.805 \\
\hline Arthralgia, n (\%) & $13(20.3 \%)$ & $15(27.2 \%)$ & - & - & 0.261 \\
\hline Rash, n (\%) & $6(9.3 \%)$ & $8(14.5 \%)$ & - & - & 0.652 \\
\hline Abdominal pain, n (\%) & $1(1.5 \%)$ & $5(9 \%)$ & $1(20 \%)$ & - & 0.090 \\
\hline Diarrhea, n (\%) & $8(12.5 \%)$ & $15(27.2 \%)$ & $1(20 \%)$ & - & 0.079 \\
\hline Vomiting, n (\%) & $10(15.6 \%)$ & $18(32.7 \%)$ & - & - & 0.082 \\
\hline $\begin{array}{l}\text { Bleeding manifestations, } \mathbf{n} \\
(\%)\end{array}$ & - & $2(3.6 \%)$ & - & - & - \\
\hline Mortality, n(\%) & - & - & - & - & \\
\hline $\begin{array}{l}\text { Thrombocytopenia } \\
<20000 / \mathrm{mm}^{3} \\
20000-50000 / \mathrm{mm}^{3} \\
50000-100000 / \mathrm{mm}^{3} \\
>100000 / \mathrm{mm}^{3}\end{array}$ & $\begin{array}{c}- \\
5 \\
16 \\
43\end{array}$ & $\begin{array}{c}- \\
14 \\
9 \\
32\end{array}$ & $\begin{array}{l}- \\
- \\
2 \\
5\end{array}$ & $\begin{array}{l}- \\
- \\
1 \\
1\end{array}$ & 0.231 \\
\hline
\end{tabular}

$\mathrm{P}$ values are calculated by Pearson Chi square Test/Fisher's Exact Test

$\mathrm{n}=$ number of patients having manifestations

No significant difference was observed on analysis of the correlation of clinical manifestations and the serotypes involved. There is no statistically significant difference between the serotypes and thrombocytopenia 
Fig.1

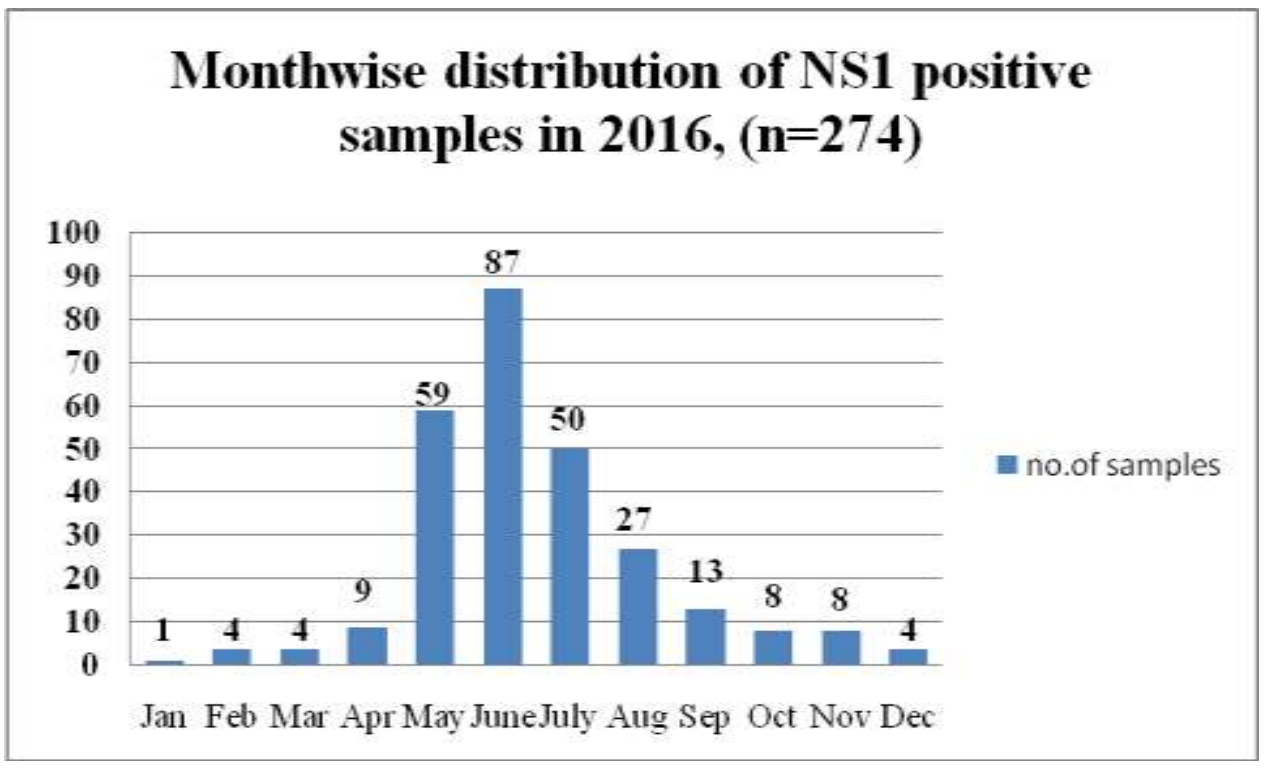

Fig.2 Gel electrophoresis report of conventional multiplex RT-PCR

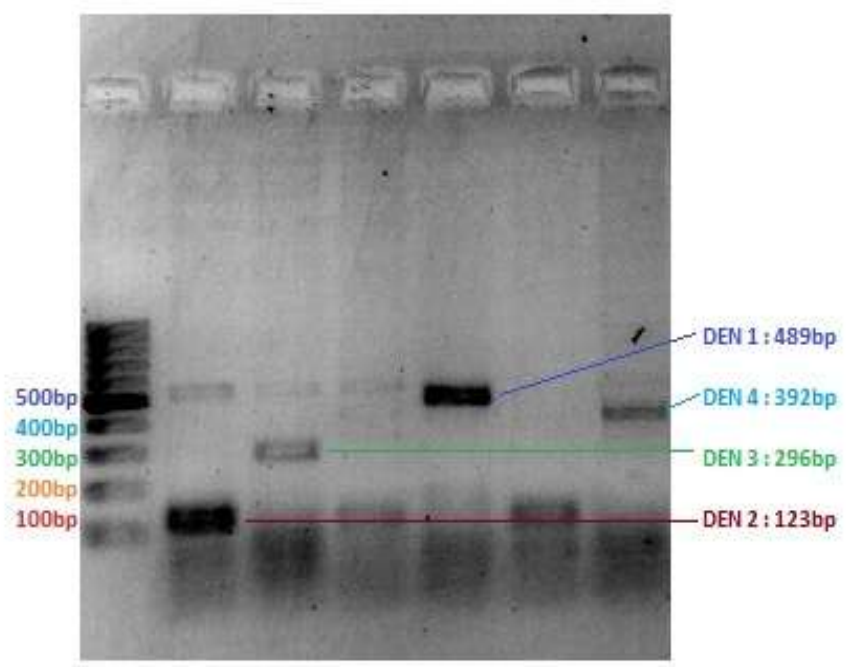

A serotypic distribution detected in 20112014 in Delhi showed an almost complete dominance of DENV-2 followed by DENV1.Concurrent infections were detected in $18 \%$ of the dengue positive cases, which were mainly caused by DENV-1 and DENV-2 revealing a high proportion of such cases in Delhi (Afreen et al., 2015).

Towards the south, in a study conducted during an outbreak in 2003 in Kanyakumari district in Tamilnadu, DENV-3 was the predominant serotype (Paramasivan et al., 2006).Dengue fever was first recorded in Kerala in Kottayam District in 1997.Kerala state, located in the southernmost tip of India, records an increasing trend of dengue fever problem since 2006 (Kalra et al., 2004). A study conducted in Ernakulam district, Kerala during 2008-2010, has reported all the serotypes in circulation, and the most predominant serotype observed was DENV-2 followed by DENV-3 (Anoop et al., 2010). 
In the present study, all the four serotypes were identified along with co-infections. DENV-1 (40.3\%) was found to be the most predominant serotype in circulation followed by DENV-2 (34.6\%) in 2016. Co-infections were mainly caused by DENV-1 and DENV$2(13.8 \%)$. No genetic data are available for DENV serotypes in Kerala prior to 2008. Due to a lack of studies in Kerala before the 2008 outbreak, we are unable to compare the serotype shifts during the outbreaks. However, with the current data available, the present study showed there is a change in circulation serotypes in the outbreaks.

The present study showed an increased incidence of gastrointestinal manifestations like abdominal pain, vomiting and diarrhea in patients infected with DENV-2 though this was not statistically significant. This correlates with a study conducted in a tertiary care hospital in Delhi over a period of 5 years (2002-2006) (Kumaria, 2010). Bleeding manifestations were identified in five cases in which 2 were by DENV-2 and the rest were observed in co-infection caused by DENV$1 \& D E N V-2$. In a retrospective study from Thailand, DENV-2 has been documented as the most frequent serotype among DHF cases (35\%) (Nisalak et al., 2003). A high incidence of gastro-intestinal manifestations like abdominal pain and diarrhea were reported in an epidemic in Kerala, 2003 (Rachel Daniel et al., 2005). Another study conducted in Noida, UP also reported the most common symptoms apart from fever and headache, were gastrointestinal symptoms like abdominal pain, vomiting, and diarrhea (Jain et al., 2015).

In the present study, one hundred and eighteen patients $(43 \%)$ had a platelet count of $<100,000 / \mathrm{mm}^{3}$. Among them, 42 patients $(35.5 \%)$ had a count $<50,000 / \mathrm{mm}^{3}$, of which 22 cases $(10.5 \%)$ were primary dengue and 20 $(30.3 \%)$ secondary dengue infection. Lower platelet count has an association with more severe dengue spectrum. This observation is correlates with studies conducted in Kanpur (Richa Giri et al., 2016). Platelet count $<50000 / \mathrm{mm}^{3}$ were more recorded in DENV-2 (14 cases-33.3\%) and DENV-1 (5 cases$11.9 \%$ ) in the present study. A study conducted in Delhi has also reported lower mean platelet count in DENV-2 cases (Kumaria, 2010). A study conducted in Bangkok, Thailand has also reported more severe clinical manifestations in DENV-2 cases (Kalayanarooj et al., 2000). None of our cases had a platelet value less than $20000 / \mathrm{mm}^{3}$.

In conclusion, this study reveals that all four serotypes were found to be co-circulating in the outbreak of 2016 in central Kerala as detected by conventional multiplex reverse transcriptase (RT)-PCR. The increasing trend of co-circulation of dengue virus serotypes suggests the transition of Kerala to a hyperendemic state from an endemic one. DENV-1 has dominated this outbreak followed by DENV-2. Majority of the coinfections are caused by DENV-1 and DENV2

\section{References}

Afreen N, Naqvi IH, Broor S, Ahmed A, Parveen S . Phylogenetic and Molecular Clock Analysis of Dengue Serotype 1 and 3 from New Delhi, India. 2015.PLoS ONE. 10(11)

Anoop. M, Issac A, Mathew T, Philip S, Kareem NA, Unnikrishnan R.2010. Genetic characterization of dengue virus serotypes causing concurrent infection in an outbreak in Ernakulam, Kerala, South India. Indian $J$ Exp Biol.48: 849-57.

Bandyopadhyay S, Jain DC, Datta KK. 1996. Reported incidence of dengue/DHF in 
India 1991-1995. Dengue Bulletin. 20:33-34.

Bharaj P, Chahar HS, Pandey A, Diddi K, Dar L, Guleria R, Sushil K Kabra SK, Broor S, 2008. Concurrent infections by all four dengue virus serotypes during an outbreak of dengue in 2006 in Delhi, India. Virology Journal. 5:1

Chakravarti A, Arora R, Luxemburger C. 2012. Fifty years of dengue in India. Trans R Soc Trop Med Hyg. 106:273282.

Daniel R, Rajamohanan, Philip AZ. 2005. A Study of Clinical Profile of Dengue Fever in Kollam, Kerala, India. Dengue Bulletin. Vol.29

Dar L, Broor S, Sengupta S, Xess I, Seth P.1999. The first major outbreak of dengue hemorrhagic fever in Delhi, India. Emerg Infect Dis. 5:589-90.

Das S S, Turbadkar D, Baveja S, Kumar C A, Ramchandran A, Randive M .2016. Seasonal Variation Influencing Dengue Transmission in Mumbai City: A Model for Outbreak Prediction. IJSR.5(7).

Dash PK, Saxena P, Abhyankar A, Bhargava R, Jana AM. 2005. Emergence of dengue virus type-3 in northern India. Southeast Asian J Trop Med Public Health. 36: 370-7.

Gibbons RV, Vaughn DW.2002. Dengue: an escalating problem. B M J. 324:156366.

Guzman MG, Halstead SB, Artsob H, Buchy P, Farrar J, Gubler DJ. 2010. Dengue: a continuing global threat. Nature Rev Microbiol. 8: S7-S16.

Guzman MG, Kouri G. 1996. Advances in dengue diagnosis. Clin Diagn Lab Immunol. 3: 621-7.

Jain P, Kuber D, Garg AK, GD Sharma, AK Agarwal. 2015. Manifestations of dengue fever: A hospital based study. Indian Academy of Clinical Medicine. $16(3 \& 4)$.
Khan E, Siddiqui J, Shakoor S, Mehraj V, Jamil B, Hasan R. 2007. Dengue outbreak in Karachi, Pakistan, 2006: experience at a tertiary care center. Trans R Soc Trop Med Hyg. 101: 1114-9.

Kumar NP, Jayakumar PR, George KT. Kamaraj, K. Krishnamoorthy, Sabesan S, Jambulingam P. 2013. Genetic characterization of dengue viruses prevalent in Kerala State. Indian Journal of Medical Microbiology. 62: 545-552

Kumar A, Rao CR, Pandit V, Shetty S, Bammigatti C, Samarasinghe CM.2010. Clinical manifestations and trend of dengue cases admitted in a tertiary care hospital, Udupi district, Karnataka. Indian J Community Med. 35(3): 386-90.

KalraN. L., Prasittisuk C.2004. Sporadic prevalence of DF/DHF in the Nilgiri and Cardamom hills of Western Ghats in South India: is it a seeding from sylvatic dengue cycle - a hypothesis. Dengue Bull. 28: 44-50.

Kalayanarooj S, Nimmannitya S. 2000. Clinical and Laboratory Presentations of Dengue Patients with Different Serotypes. Dengue bulletin. Vol 24.

Kumaria R. 2010. Correlation of disease spectrum among four Dengue serotypes: a five years hospital based study from India. Braz J Infect Dis. 14(2):141-146

Kuno G, Gomez I, Gubler DJ. 1991. An ELISA procedure for the diagnosis of dengue infections. J Virol Methods. 33: 101-13.

Lal M, Aggarwal A, Oberoi A. 2007. Dengue Fever- An emerging viral fever in Ludhiana, North India. Indian J Public Health. 51: 198-9.

Lanciotti RS, Calisher CH, Gubler DJ, Chang GJ, Vorndam AV. 1992. Rapid detection and typing of dengue viruses 
from clinical samples by using reverse transcriptase-polymerase chain reaction. J Clin Microbiol. 30: 545-51.

Lall R, Dhanda V. 1996. Dengue haemorrahagic fever and the dengue shock syndrome in India. Natl Med J India. 9: 20.

Mustafa MS, Rastogi V, Jain S, Gupta V.2015. Discovery of fifth serotype of dengue virus (DENV-5): A new public health dilemma in dengue control. Medical journal armed forces India.7: 67-70

McBride WJH and HelleBielefeldt-Ohmann. 2000. Dengue viral infections; pathogenesis and epidemiology. Microbes and Infection. 2(9): 1041-50.

Nisalak A, Endy TP, Nimmanniya, kalayanarooj S, Thisayakorn U, Scott RM, Burke DS, Hoke CH, Innis BL, Vaughn DW. 2003. Serotype-specific dengue virus circulation and dengue disease in Bangkok, Thailand from 1973-1979. Am J Trop Med Hyg. 68:191-202.

Paramasivan R, Thenmozhi V, Hiriyan J, Dhananjeyan K, Tyagi B, Dash AP. 2006. Serological and entomological investigations of an outbreak of dengue fever in certain rural areas of Kanyakumari district, Tamil Nadu. Indian J Med Res. 123: 697-701.

Rastogi M, Sharma N, Singh SK. 2016. Flavivirus NS1: a multifaceted enigmatic viral protein. Virology Journal. 13:131.

Richa Giri, Agarwal K, Verma S, Verma R, Lipok. 2016. A study to correlate level of thrombocytopenia with dengue seropositive patients and frequency of bleeding pattern. Sch. J. App. Med. Sci. 4(1C):214-218

Satish N, Vijaykumar TS, Abraham P, Sridharan G. 2003. Dengue fever: it's laboratory diagnosis with special emphasis on IgM detection. WHO Dengue Bull. 27: 116-125.

Sreekumar E, Isaac A, Nair S, Hariharan R, Janki M B, Arathy D S. 2010. Genetic characterization of 2006-2008 isolates of Chikunguya virus from Kerala, South India, by whole genome sequence analysis. Virus genes. 40(14).

Tyagi BK, Hiriyan J, Samuel P, Tewari SC, Paramasivan R. 2006. Dengue in Kerala: A critical review. ICMR Bull. 6:13-30

Weaver SC, Vasilakis N. 2009. Molecular evolution of dengue viruses: contributions of phylogenetics to understanding the history and epidemiology of the preeminent arboviral disease. Infect Genet Evol. 9: 523-540.

World Health Organisation. 2009. Dengue: Guidelines for Diagnosis, Treatment, Prevention and Control, New Edition, World Health Organization and TDR for research on diseases of poverty.

\section{How to cite this article:}

Kavitha R. Nair, Seema Oommen, O.A. Jagan and Vidya Pai. 2019. Detection of Circulating Dengue Virus Serotypes in a Tertiary Care Centre in Central Kerala, 2016. Int.J.Curr.Microbiol.App.Sci. 8(01): 2669-2678. doi: https://doi.org/10.20546/ijcmas.2019.801.281 